\title{
ISOMETRIES AND APPROXIMATE ISOMETRIES
}

\section{THEMISTOCLES M. RASSIAS}

\author{
(Received 15 October 1999)
}

\begin{abstract}
Some properties of isometric mappings as well as approximate isometries are studied.
\end{abstract}

2000 Mathematics Subject Classification. Primary $46 B 04$.

1. Isometry and linearity. Mazur and Ulam [17] proved the following well-known result concerning isometries, that is, transformations which preserve distances.

THEOREM 1.1. Given two real normed vector spaces $X$ and $Y$, let $U$ be a surjective mapping from $X$ onto $Y$ such that $\|U(x)-U(y)\|=\|x-y\|$ for all $x$ and $y$ in $X$. Then the mapping $x \mapsto U(x)-U(0)$ is linear.

Since continuity is implied by isometry, the proof of this theorem consists of showing that $U(x)-U(0)$ is additive, and the additivity of this mapping will follow if we can prove that $U$ satisfies Jensen's equation:

$$
U\left(\frac{x}{2}+\frac{y}{2}\right)=\frac{U(x)}{2}+\frac{U(y)}{2} \quad \forall x, y \in X .
$$

In general this is not easy. However, for the special case in which the space $Y$ is strictly convex, the proof is simple (see Baker [1]). A (real) normed space is called strictly convex if, for each pair of its nonzero elements $y, z$ such that $\|y+z\|=\|y\|+\|z\|$, it follows that $y=c z$ for some real number $c>0$. When $Y$ is strictly convex, it is easy to show that the unique solution to the two equations

$$
\left\|m-y_{1}\right\|=\left\|y_{2}-m\right\|, \quad\left\|m-y_{1}\right\|+\left\|y_{2}-m\right\|=\left\|y_{2}-y_{1}\right\|
$$

is $m=\left(y_{1} / 2\right)+\left(y_{2} / 2\right)$. Following Baker [1], we find that, from the second equation, since $Y$ is strictly convex, $m-y_{1}=c\left(y_{2}-m\right)$ with $c>0$. From the first equation of (1.2), it follows that $c=1$ and $m=\left(y_{1} / 2\right)+\left(y_{2} / 2\right)$. Now, for any given pair $x_{1}, x_{2}$ in $X$, let $h=\left(x_{1}+x_{2}\right) / 2$ and put $y_{1}=U\left(x_{1}\right), y_{2}=U\left(x_{2}\right)$. Clearly, we have

$$
\left\|h-x_{1}\right\|=\left\|x_{2}-h\right\|, \quad\left\|h-x_{1}\right\|+\left\|x_{2}-h\right\|=\left\|x_{2}-x_{1}\right\| .
$$

Since $U$ is an isometry, it follows that

$$
\left\|U(h)-y_{1}\right\|=\left\|y_{2}-U(h)\right\|, \quad\left\|U(h)-y_{1}\right\|+\left\|y_{2}-U(h)\right\|=\left\|y_{2}-y_{1}\right\| .
$$

Hence, $m=U(h)$ is the unique solution of (1.2), so that $U$ is a solution of Jensen's equation (1.1). It follows that $U(x)-U(0)$ is additive (cf. Aczél [9, page 43]). 
In this strictly convex case, it was not necessary to assume that the mapping $U$ was surjective. However, in general, the Mazur-Ulam Theorem 1.1 fails without this assumption. Among counterexamples in the literature which show this, we cite the following from Figiel [10]. Let $Y$ denote the space of real number pairs $y=\left(y_{1}, y_{2}\right)$ with the norm $\|y\|=\max \left[\left|y_{1}\right|,\left|y_{2}\right|\right]$, and consider the mapping $T: \mathbb{R} \rightarrow Y$ given by $T(s)=(s, \sin (s)), s$ in $\mathbb{R}$. Then, for $s_{1}$ and $s_{2}$ in $\mathbb{R}$, we have

$$
\left\|T\left(s_{2}\right)-T\left(s_{1}\right)\right\|=\max \left\{\left|s_{2}-s_{1}\right|,\left|\sin s_{2}-\sin s_{1}\right|\right\} .
$$

Now

$$
\left|\sin \left(s_{2}\right)-\sin \left(s_{1}\right)\right|=\left|\int_{s_{1}}^{s_{2}}(\cos x) d x\right| \leq\left|s_{2}-s_{1}\right|
$$

so that $T$ is an isometry but clearly is not additive. Of course, this space $Y$ is far from being strictly convex. Indeed, if we take $y_{1}=(0,0)$ and $y_{2}=(2,0)$ in $(1.2)$, we see that the solutions $m$ to these equations form an infinite set, namely the line segment joining the points $(1,-1)$ and $(1,1)$.

In their proof of Theorem 1.1, Mazur and Ulam found a way to produce a metric characterization of the midpoint of the segment joining two points $y_{1}$ and $y_{2}$ of an arbitrary normed vector space $Y$. They did this by constructing a sequence of sets $H_{n}$ in $Y, n=1,2,3, \ldots$, defined recursively by

$$
\begin{gathered}
H_{1}=\left\{y \in Y:\left\|y-y_{1}\right\|=\left\|y-y_{2}\right\|=\frac{\left\|y_{1}-y_{2}\right\|}{2}\right\}, \\
H_{n}=\left\{y \in H_{n-1}:\|y-z\| \leq \frac{\delta\left(H_{n-1}\right)}{2} \forall z \in H_{n-1}\right\} \quad \text { for } n \geq 2,
\end{gathered}
$$

where $\delta\left(H_{n-1}\right)$ denotes the diameter of the set $H_{n-1}$. The intersection of all the sets $H_{n}$ is called the metric center of $y_{1}$ and $y_{2}$. They proved that it consists of a single point $\left(y_{1} / 2\right)+\left(y_{2} / 2\right)$.

Vogt [24] generalized Theorem 1.1 by considering mappings which preserve "equality of distance." These are mappings $f: X \rightarrow Y$ between normed vector spaces such that there exists a function $p:[0, \infty) \rightarrow[0, \infty)$ with $\|f(x)-f(y)\|=p(\|x-y\|)$. Such mappings were studied by Schoenberg [20] and by von Neumann and Schoenberg [25]. In Vogt's approach, a basic result was the following theorem concerning isometries in metric spaces.

THEOREM 1.2 (Vogt [24]). Let $(M, d)$ be a bounded metric space. Suppose that there is an element $m \in M$, a surjective isometry $g: M \rightarrow M$ and a constant $k>1$ such that $d(g x, x) \geq k d(m, x)$ for all $x$ in $M$. Then $m$ is a fixed point for every surjective isometry $h: M \rightarrow M$.

Proof. Let $h: M \rightarrow M$ be any surjective isometry. Since surjective isometries are injective, $g^{-1}$ and $h^{-1}$ exist and $g, h, g^{-1}, h^{-1}$ are bijective isometries of $M$ together with finite compositions of them. We define a sequence of isometries $g_{n}: M \rightarrow M$ and a sequence of elements $m_{n}(n \in N)$ defined recursively by

$$
\begin{aligned}
& g_{1}=g, \quad m_{1}=m, \\
& g_{2}=h g h^{-1}, \quad m_{2}=h m, \\
& g_{n+1}=g_{n-1} g_{n}\left(g_{n-1}\right)^{-1}, \quad m_{n+1}=g_{n-1} m_{n}, \quad n \geq 2 .
\end{aligned}
$$


Clearly, each $g_{n}$ is invertible on $M$. We now prove by induction that

$$
d\left(g_{n} x, x\right) \geq k d\left(m_{n}, x\right) \quad \forall x \in M .
$$

By hypothesis, (1.9) is true for $n=1$. When $n=2, d\left(m_{2}, x\right)=d(h m, x)=d\left(m, h^{-1} x\right)$ since $h^{-1}$ is an isometry. Also,

$$
d\left(g_{2} x, x\right)=d\left(h g h^{-1} x, x\right)=d\left(g h^{-1} x, h^{-1} x\right) \geq k d\left(m, h^{-1} x\right)=k d\left(m_{2}, x\right),
$$

which demonstrates (1.9) for $n=2$. Make the induction assumption that $d\left(g_{n} x, x\right) \geq$ $k d\left(m_{n}, x\right)$ when $n \geq 2$. Then

$$
\begin{aligned}
d\left(g_{n+1} x, x\right) & =d\left(g_{n-1} g_{n} g_{n-1}^{-1} x, x\right)=d\left(g_{n} g_{n-1}^{-1} x, g_{n-1}^{-1} x\right) \\
& \geq k d\left(m_{n}, g_{n-1}^{-1} x\right)=k d\left(g_{n-1} m_{n}, x\right)=k d\left(m_{n+1}, x\right),
\end{aligned}
$$

so the induction proof is complete and (1.9) is true for all $n \in N$.

If we put $x=m_{n+1}$ in (1.9), we find that

$$
d\left(m_{n+2}, m_{n+1}\right)=d\left(g_{n} m_{n+1}, m_{n+1}\right) \geq k d\left(m_{n+1}, m_{n}\right) .
$$

By another induction, we obtain $d\left(m_{n+2}, m_{n+1}\right) \geq k^{n} d\left(m_{2}, m_{1}\right)$ for all $n \geq 1$. Since the metric space $M$ is bounded there exists a positive number $B$ such that $B>$ $d\left(m_{n+2}, m_{n+1}\right)$ for all $n \geq 1$. Hence $\left(B / k^{n}\right) \geq d\left(m_{2}, m_{1}\right)$ for all $n \in N$. By hypothesis, $k>1$, and it follows that $d\left(m_{2}, m_{1}\right)=d(h m, m)=0$, so that $m$ is a fixed point of $h$.

Proof OF Theorem 1.1. Instead of using the original proof of Theorem 1.1 due to Mazur and Ulam, we shall rely on Vogt's method. This will "set the stage" for later developments when we deal with approximate isometries.

Put $f(x)=U(x)-U(0)$, so that $f: X \rightarrow Y$ is a surjective isometry with $f(0)=0$. Given a fixed element $x$ in $X$, define $M$ as the set

$$
M=\{y \in Y:\|y\|=\|2 f(x)-y\| \leq 2\|f(x)\|\} .
$$

Put $m=f(x)$ and let $g: M \rightarrow M$ be given by $g(y)=2 f(x)-y$. Then we see that

(i) $M$ is a bounded metric space, with $d(\cdot, \cdot)=\|\cdot-\cdot\|$,

(ii) $m=f(x)$ belongs to $M$,

(iii) $g$ is an isometry from $M$ onto $M$, and $g(M)=M$ since $g$ is its own inverse,

(iv) $d(g y, y) \geq k d(m, y)$ for all $y$ in $M$ with $k=2$.

Property (iv) is true because

$$
d(g y, y)=\|g(y)-y\|=\|2 f(x)-2 y\|=2\|f(x)-y\|=2 d(m, y) .
$$

By Theorem 1.2 and properties (i)-(iv), we conclude that $m$ is a fixed point of every surjective isometry $h$ of $M$. In particular, we put $x_{0}=f^{-1}(2 f(x))$ and define $h: M \rightarrow M$ by $h(y)=f\left(x_{0}-f^{-1}(y)\right)$.

(v) $h$ is a surjective isometry from $M$ onto $M$. 
With $f\left(x_{1}\right)=y_{1}$ in $M$, we have

$$
\begin{aligned}
\left\|2 f(x)-h\left(y_{1}\right)\right\| & =\left\|2 f(x)-f\left(x_{0}-x_{1}\right)\right\|=\left\|f\left(x_{0}\right)-f\left(x_{0}-x_{1}\right)\right\|=\left\|x_{1}\right\| \\
& =\left\|f\left(x_{1}\right)\right\|=\left\|y_{1}\right\|=\left\|2 f(x)-y_{1}\right\|=\left\|f\left(x_{0}\right)-f\left(x_{1}\right)\right\| \\
& =\left\|f\left(x_{0}-x_{1}\right)\right\|=\left\|f\left(x_{0}-f^{-1}\left(y_{1}\right)\right)\right\|=\left\|h\left(y_{1}\right)\right\| .
\end{aligned}
$$

Thus, $h\left(y_{1}\right)$ is in $M$. But $h$ is its own inverse, so $h(M)=M$.

Now, by Theorem 1.2, $m$ is a fixed point of $h$. Thus, we have

$$
\begin{aligned}
f(x) & =m=h(m)=f\left(x_{0}-f^{-1}(m)\right)=f\left(x_{0}-x\right), \\
0 & =\left\|f(x)-f\left(x_{0}-x\right)\right\|=\left\|x-\left(x_{0}-x\right)\right\|=\left\|2 x-x_{0}\right\|=\left\|f(2 x)-f\left(x_{0}\right)\right\| \\
& =\|f(2 x)-2 f(x)\| .
\end{aligned}
$$

Thus, we have proved that

(vi) $f(2 x)=2 f(x)$ for all $x \in X$.

For any given $y$ in $X$, put $f_{y}(x)=f(x+y)-f(y)$ for all $x \in X$. Then $f_{y}: X \rightarrow Y$ is a surjective isometry and $f_{y}(0)=0$, so that $f_{y}$ satisfies (vi). For any pair $x, y$ in $X$, we have

$$
\begin{aligned}
f(x)-f(y) & =f[(x-y)+y]-f(y)=f_{y}(x-y) \\
& =2 f_{y}\left(\frac{x-y}{2}\right)=2\left(f\left(\frac{x-y}{2}+y\right)-f(y)\right) .
\end{aligned}
$$

Thus,

$$
f(x)+f(y)=2 f\left(\frac{x-y}{2}+y\right)=2 f\left(\frac{x+y}{2}\right)=f(x+y) .
$$

COMMENTS: GENERALIZATIONS OF THE MAZUR-Ulam THEOREM. As indicated above, Vogt used his method, that was used above to prove Theorem 1.1, together with other considerations in order to prove the following generalization.

THEOREM 1.3. Given real normed spaces $X$ and $Y$, let $f: X \rightarrow Y$ with $f(0)=0$ be a surjective mapping which preserves equality of distance. Then $f$ is linear and $f=\beta U$, where $\beta \neq 0$ is a real number and $U: X \rightarrow Y$ is a surjective isometry, providing that the dimension of $X$ is greater than one.

Vogt also gave an example to show that this theorem fails when $X$ is one-dimensional.

We have seen that, in general, the isometry must be surjective in order to be linear. However, Figiel [10] proved the following result which amounts to another generalization of the Mazur-Ulam theorem.

THEOREM 1.4. Given real normed vector spaces $X$ and $Y$, let $\varphi: X \rightarrow Y$ be an isometric embedding of $X$ into $Y$. Then there exists a linear (not necessarily continuous) mapping $F: Y \rightarrow X$ such that $F \circ \varphi$ is the identity on $X$ and the restriction of $F$ to the linear span of $\varphi(X)$ is of norm one.

Various other generalizations or variations of the Mazur-Ulam theorem may be found in the literature. Charzyński [7] proved the theorem for metric vector spaces 
of the same finite dimension. Day [8, pages 110-111], demonstrated a version of the theorem involving semi-norms (instead of norms or metrics) in locally convex topological vector spaces. Rolewicz [19] proved the theorem for a class of metric vector spaces which are locally bounded and such that the function $\varphi(t)=d(t x, 0)$ is concave in the real $t$ for each fixed $x$ in the space. An example of such a space is the set of sequences $x=\left(x_{1}, x_{2}, \ldots\right)$ such that $d(x, 0)=\sum_{k=1}^{\infty}\left|x_{k}\right|^{p}$ converges, with $0<p<1$. Wobst [26] generalized the results of Charzyński and of Rolewicz.

To illustrate what can happen when an isometry is not surjective, we again turn to Baker [1] who gives two examples of isometries which are not affine. The first example is from Fischer and Muszély [11]: let $Y$ be a real normed vector space which is not strictly convex. Thus, we may choose $a, b$ in $Y$ so that $a$ and $b$ are linearly independent and satisfy $\|a\|=\|b\|=1$ and $\|a+b\|=\|a\|+\|b\|$. Let $f: \mathbb{R} \rightarrow Y$ be defined by

$$
f(x)= \begin{cases}x a & \text { if } x \leq 1, \\ a+(x-1) b & \text { if } x>1 .\end{cases}
$$

Using Baker's lemma, which states that $\|a+b\|=\|a\|+\|b\|$ implies that $\|s a+t b\|=$ $s\|a\|+t\|b\|$ when $s \geq 0$ and $t \geq 0$, it is easy to see that $f$ is an isometry. Also, $f(0)=0$ and $f$ is nonlinear.

The second example was constructed by Baker in answer to a problem of Chernoff (Advanced Problem 5688, Amer. Math. Monthly 76 (1969), 835). It shows that an isometry can be not only nonlinear but also homogeneous of degree one, and is defined as follows: let $Y$ be the set $\mathbb{R}^{3}$ but with the metric given by the norm $\|(x, y, z)\|=$ $\max \left[\sqrt{x^{2}+y^{2}},|z|\right]$. Let $f: \mathbb{R}^{2} \rightarrow Y$ be given by $f(x, y)=(x, y, g(x, y))$, with

$$
g(x, y)= \begin{cases}y & \text { if } 0 \leq y \leq x \text { or } x \leq y \leq 0 \\ x & \text { if } 0 \leq x \leq y \text { or } y \leq x \leq 0 \\ 0 & \text { otherwise }\end{cases}
$$

Clearly, $g$ satisfies $g(t x, t y)=\operatorname{tg}(x, y)$ for real $t, x, y$, and it is easily seen that $g$ is not linear. Also it can be shown that $|g(x, y)-g(u, v)| \leq \sqrt{(x-u)^{2}+(y-v)^{2}}$. Thus, $f: \mathbb{R}^{2} \rightarrow Y$ is an isometry which is nonlinear and homogeneous.

2. Approximate isometries: special cases. By an $\varepsilon$-isometry of one metric space $E$ into another $E^{\prime}$, we mean a mapping $T: E \rightarrow E^{\prime}$ which changes distances between elements of $E$ by at most $\varepsilon$ for some fixed $\varepsilon>0$, that is,

$$
\left|d^{\prime}(T(x), T(y))-d(x, y)\right| \leq \varepsilon \quad \forall x, y \in E,
$$

where $d$ and $d^{\prime}$ are the metrics for $E$ and $E^{\prime}$, respectively. The stability question here is: given $\eta>0$, does there exist $\varepsilon>0$ and an isometry $U: E \rightarrow E^{\prime}$ such that $d^{\prime}(T(x), U(x))<\eta$ for all $x$ in $E$ ? In the case, where $E$ is a real Hilbert space and $E=E^{\prime}$, an answer was given in the affirmative by Hyers and Ulam [14], providing that $T$ was surjective. Using the geometry of Hilbert space, they showed that, if $T: E \rightarrow E$ is an $\varepsilon$-isometry with $T(0)=0$, then

$$
\left\|T\left(\frac{x}{2}\right)-\frac{T(x)}{2}\right\| \leq \sqrt{2 \varepsilon\|x\|}+2 \varepsilon, \quad x \in E .
$$


They proved that the limit

$$
U(x)=\lim _{n \rightarrow \infty} \frac{T\left(2^{n} x\right)}{2^{n}}
$$

exists for all $x$ in $E$ and that $U$ is an isometry. Finally, it was shown that, if the $\varepsilon$-isometry $T$ is surjective, then $U$ is also surjective and the inequality

$$
\|T(x)-U(x)\|<k \varepsilon
$$

is satisfied for all $x$ in $E$, with $k=10$.

This theorem was generalized by Bourgin [3], who obtained the results (2.2b) and (2.3) with $k=12$ for an $\varepsilon$-isometry $T$ of a Banach space $E_{1}$ onto a Banach space $E_{2}$, where $E_{2}$ belongs to a class of uniformly convex spaces which includes the spaces $L_{p}(0,1)$ for $1<p<\infty$.

Hyers and Ulam [15] studied $\varepsilon$-isometries for spaces of continuous functions. According to a famous theorem of Banach [2, page 170], two compact metric spaces $S_{1}, S_{2}$ are homeomorphic if and only if the spaces $C\left(S_{1}\right)$ and $C\left(S_{2}\right)$ of real continuous functions defined on $S_{1}$ and $S_{2}$ are isometric. If $h$ is a homeomorphism from $S_{1}$ onto $S_{2}$, it is easily seen that $C\left(S_{1}\right)$ and $C\left(S_{2}\right)$ are isometric. For, if $\varphi \in C\left(S_{1}\right)$, we define $\psi(s)=\varphi\left(h^{-1}(s)\right)$ with $s \in S_{2}$. Then $\psi=U(\varphi)$ is an isometry from $C\left(S_{1}\right)$ onto $C\left(S_{2}\right)$. The hard part is to obtain the homeomorphism from the isometry.

Banach's theorem suggested a method of attacking the problem of stability for $\varepsilon$ isometries between spaces of continuous functions. Instead of using Banach's idea of peak functions, a different method of relating the $\varepsilon$-isometry to the correspondence between the points of $S_{1}$ and $S_{2}$ was used, by dealing with hyperplanes of functions having the same value at a given point. We shall outline the results without giving the details of the proofs.

LEMMA 2.1. Let $S_{1}$ and $S_{2}$ be compact metric spaces, and let $T: C\left(S_{1}\right) \rightarrow C\left(S_{2}\right)$ be a continuous $\varepsilon$-isometry. With $p$ in $S_{1}, q$ in $S_{2}$ and real numbers $b, c, h, k>0$, define the sets

$$
\begin{aligned}
M_{1}(p, b, h) & =\left\{\varphi \in C\left(S_{1}\right):|\varphi(p)-b| \leq h\right\}, \\
M_{2}(q, c, k) & =\left\{\psi \in C\left(S_{2}\right):|\psi(q)-c| \leq k\right\} .
\end{aligned}
$$

Then, for each point $p$ of $S_{1}$, each $b$ in $\mathbb{R}$ and each $a \geq 0$, there exists a point $q$ in $S_{2}$ and $c$ in $\mathbb{R}$ such that

$$
T\left(M_{1}(p, b, a)\right) \subset M_{2}\left(q, c, a+\frac{3 \varepsilon}{2}\right) .
$$

This lemma was the first step in establishing a correspondence between points $p$ of $S_{1}$ and $q$ of $S_{2}$. A corollary was proved to show that, when $T$ is a surjective homeomorphism as well as an $\varepsilon$-isometry of $C\left(S_{1}\right)$ onto $C\left(S_{2}\right)$, then the point $q$ of $C\left(S_{2}\right)$ is uniquely determined by the point $p$ of $S_{1}$ and is independent of the choice of the parameters $b$ and $a$. Finally, the following stability result was proved.

THEOREM 2.2. Let $S_{1}$ and $S_{2}$ be compact metric spaces. If $T$ is a homeomorphic mapping of $C\left(S_{1}\right)$ onto $C\left(S_{2}\right)$ which is also an $\varepsilon$-isometry, then there exists an isometry $U: C\left(S_{1}\right) \rightarrow C\left(S_{2}\right)$ such that $\|U(f)-T(f)\| \leq 21 \varepsilon$ for all $f$ in $C\left(S_{1}\right)$. 
COROLLARY 2.3. Under the hypotheses of Theorem 2.2, the underlying spaces $S_{1}$ and $S_{2}$ are homeomorphic.

REMARK 2.4. Incidentally, Corollary 2.3 shows that we have a generalization of Banach's theorem, where isometry is replaced by $\varepsilon$-isometry.

Stone [22] generalized Banach's theorem by replacing compact metric spaces with completely regular topological spaces, and this result is now known as the BanachStone theorem (cf. [8, page 86]).

Bourgin [4] obtained a significant generalization of Theorem 2.2 by adapting the basic idea of the proof outlined above to the more general case, with the following principal result. Here $C\left(S_{j}\right)$ denotes the space of bounded continuous functions on $S_{j}, j=1,2$.

THEOREM 2.5. Let $S_{1}$ and $S_{2}$ be completely regular Hausdorff spaces and let $T$ : $C\left(S_{1}\right) \rightarrow C\left(S_{2}\right)$ be a surjective $\varepsilon$-isometry with $T(0)=0$. Then there exists a linear isometry $U: C\left(S_{1}\right) \rightarrow C\left(S_{2}\right)$ for which $\|T(f)-U(f)\| \leq 10 \varepsilon$, for all $f \in C\left(S_{1}\right)$.

NOTE. No continuity conditions were needed in Bourgin's theorem on the $\varepsilon$-isometry $T$, in contrast to the conditions of Theorem 2.2. His theorem also results in a generalization of the Banach-Stone theorem, again by replacing isometries by $\varepsilon$-isometries. More explicitly, he defined a $\mu$-ideal as the subset of $C\left(S_{1}\right)$ given by $J(p, \mu)=\{\varphi \in$ $\left.C\left(S_{1}\right):|\varphi(p)|<\mu\right\}$, and similarly for $C\left(S_{2}\right)$, and proved the following theorem.

THEOREM 2.6. Let $S_{1}$ and $S_{2}$ be compact Hausdorff spaces. If $H$ is a possibly multiplevalued mapping of $C\left(S_{1}\right)$ onto $C\left(S_{2}\right)$ and yields a correspondence of $\mu$-ideals of either algebra to $(\mu+p)$-ideals of the other, then $S_{1}$ and $S_{2}$ are homeomorphic.

3. Approximate isometries: the general case. The problem of stability of isometry for mappings between arbitrary real normed vector spaces remained open for many years after the work on special cases discussed in the previous section. In the present section, all normed spaces are assumed to be real.

The study of $\varepsilon$-isometries of Banach spaces was revived by Bourgin [6] and Bourgin [5], Omladič and Šemrl [18]. Gruber [13] demonstrated the stability of surjective isometries between all finite dimensional normed vector spaces. In addition, these authors obtained information concerning $\varepsilon$-isometries between arbitrary normed vector spaces which will be indispensible in the proof of the general case to be given below. In particular, the following lemma is a slight modification of Bourgin [6, Lemma 2.8].

LEMMA 3.1 (R. D. Bourgin and P. L. Renz). Let $X$ and $Y$ be normed vector spaces, $\eta$ and $\delta \in \mathbb{R}_{+}$, and let $T: X \rightarrow Y$ be an $\eta$-isometry with $T(0)=0$. Then there exists $a$ continuous $(2 \delta+3 \eta)$-isometry $T^{\prime}: X \rightarrow Y$ such that $\left\|T^{\prime}(x)-T(x)\right\|<\eta+\delta$ for each $x \in X$.

Proof. Consider all subsets of $X$ such that the distance between each pair of distinct elements is at least $\delta / 2$. By Zorn's lemma, there exists a maximal such collection which will be denoted by $\left\{x_{\gamma}: \gamma \in \Gamma\right\}$. Then, for any $x \in X$, there is a $\gamma \in \Gamma$ with $\left\|x-x_{y}\right\| \leq \delta / 2$. Let $B_{y}$ denote the ball $\left\{x \in X:\left\|x-x_{\gamma}\right\|<\delta\right\}$. 
Then $\left\{B_{\gamma}: \gamma \in \Gamma\right\}$ is an open cover of $X$. Hence, there is a partition of unity $\left\{f_{\xi}\right.$ : $\xi \in \Xi\}$ subordinate to $\left\{B_{\gamma}: \gamma \in \Gamma\right\}$. For each $\xi \in \Xi$, pick any $\gamma \in \Gamma$ such that $\{y \in X$ : $\left.f_{\xi}(y) \neq 0\right\} \subset B_{\gamma}$ and denote this $\gamma$ by $\gamma(\xi)$. Thus, $\left\{y \in X: f_{\xi}(y) \neq 0\right\} \subset B_{\gamma(\xi)}$ for each $\xi \in \Xi$. Define $T^{\prime}: X \rightarrow Y$ by the formula $T^{\prime}(x)=\sum_{\xi \in \Xi} f_{\xi}(x) T\left(x_{\gamma(\xi)}\right)$ for each $x \in X$. Thus the function $T^{\prime}$ is well defined and continuous. Also, for $x \in X$,

$$
\begin{aligned}
\left\|T^{\prime}(x)-T(x)\right\| & =\left\|\sum_{\xi \in \Xi} f_{\xi}(x)\left(T\left(x_{\gamma(\xi)}\right)-T(x)\right)\right\| \\
& \leq \sum_{\xi \in \Xi} f_{\xi}(x)\left\|T\left(x_{\gamma(\xi)}\right)-T(x)\right\| \\
& \leq \sum_{\xi \in \Xi} f_{\xi}(x)\left(\left\|x_{\gamma(\xi)}-x\right\|+\eta\right) .
\end{aligned}
$$

Now, if $f_{\xi}(x) \neq 0$, then $\left\|x-x_{\gamma(\xi)}\right\|<\delta$, so that

$$
\left\|T^{\prime}(x)-T(x)\right\| \leq \sum_{\xi \in \Xi} f_{\xi}(x)(\delta+\eta)=\delta+\eta \quad \forall x \in X .
$$

It follows that $T^{\prime}$ is a continuous $(2 \delta+3 \eta)$-isometry.

Bourgin [6] also introduced the following concept which also will be useful later.

DEFINITION 3.2. Given $\delta>0$, a function $f: X \rightarrow Y$ between normed vector spaces is said to be $\delta$-onto if, for each $y \in Y$, there is a point $x \in X$ for which $\|f(x)-Y\| \leq \delta$.

Gruber [13] obtained an elegant and definitive result, as follows.

THEOREM 3.3. Let $X$ and $Y$ be normed spaces. Given $\varepsilon>0$, suppose that $T: X \rightarrow Y$ is a surjective $\varepsilon$-isometry while $U: X \rightarrow Y$ is an isometry such that $T(0)=U(0)=0$. If $\|T(x)-U(x)\| /\|x\| \rightarrow 0$ uniformly as $\|x\| \rightarrow \infty$ uniformly, then $U$ is a surjective linear isometry and

$$
\|T(x)-U(x)\| \leq 5 \varepsilon \quad \forall x \in X .
$$

The proof requires several lemmas subject to the hypotheses of Theorem 3.3.

LEMMA 3.4. $U$ is a surjective isometry.

Proof. From the hypotheses of the theorem, we have $\|x\| /\|T x\| \leq 1+\varepsilon$ for large $\|x\|$, and

$$
\frac{\|T(x)-U(x)\|}{\|T(x)\|}=\left(\frac{\|T(x)-U(x)\|}{\|x\|}\right)\left(\frac{\|x\|}{\|T(x)\|}\right) \leq\left(\frac{\|T(x)-U(x)\|}{\|x\|}\right)(1+\varepsilon),
$$

so that

$$
\left(\frac{\|T(x)-U(x)\|}{\|T(x)\|}\right) \rightarrow 0
$$

uniformly as $\|T(x)\| \rightarrow \infty$ uniformly. Hence, $Y$ is the closure of the linear hull of $U(X)$. Now we use Theorem 1.4 above to deduce the existence of a map $J: Y \rightarrow X$, where $J$ is linear and such that $J \circ U=\mathrm{id}_{X}=$ identity on $X$, and $\|J\|=1$. We must show that $J$ is bijective. Assume that $J$ is not injective. Then, because $J$ is linear, there exists $y \neq 0$ in $Y$ with $J(y)=0$, so that $J(\beta y)=0$ for all $\beta \in \mathbb{R}_{+}$. As was shown above, 
$\|T(x)-U(x)\| /\|T(x)\| \rightarrow 0$ uniformly as $\|T(x)\| \rightarrow \infty$ uniformly. Therefore, we may choose for each $\beta \in \mathbb{R}_{+}$a point $x_{\beta} \in X$ such that $\left\|\beta y-U\left(x_{\beta}\right)\right\| /\|\beta y\|$ and so also $\left\|\beta y-U\left(x_{\beta}\right)\right\| / \beta \rightarrow 0$ as $\beta \rightarrow \infty$. Recalling that $J \circ U=\operatorname{id}_{X}$ and $\|J\|=1$, we have

$$
\begin{aligned}
0 & =\|J(\beta y)\| \geq\left\|J\left(U\left(x_{\beta}\right)\right)\right\|-\left\|J(\beta y)-J\left(U\left(x_{\beta}\right)\right)\right\| \geq\left\|x_{\beta}\right\|-\left\|\beta y-U\left(x_{\beta}\right)\right\| \\
& =\left\|U\left(x_{\beta}\right)\right\|-\left\|\beta y-U\left(x_{\beta}\right)\right\| \geq\|\beta y\|-2\left\|\beta y-U\left(x_{\beta}\right)\right\|,
\end{aligned}
$$

so that

$$
0 \geq\|y\|-\frac{2\left\|\beta y-U\left(x_{\beta}\right)\right\|}{\beta} \rightarrow\|y\| \quad \text { as } \beta \longrightarrow \infty .
$$

This contradiction proves that $J$ is injective. Since $J \circ U=\mathrm{id}_{X}, J$ is also surjective and thus bijective.

LEMMA 3.5. The isometry $U: X \rightarrow Y$ is given by the formula

$$
U(x)=\lim 2^{-n} T\left(2^{n} x\right), \quad x \in X .
$$

Proof. By the hypotheses of the theorem, given $\delta>0$, there exists $M_{\delta}>0$ such that $\|T(z)-U(z)\| \leq \delta\|z\|$ for all $z \in X$ with $\|z\|>M_{\delta}$. For $x=0$, equation (3.8) holds. For a given $x \neq 0$ in $X$, choose $m$ so large that $\left\|2^{m} x\right\| \geq M_{\delta}$. Now $U$ is linear, so, when $n \geq m$, we have

$$
\left\|T\left(2^{n} x\right)-2^{n} U(x)\right\| \leq \delta\left\|2^{n} x\right\|, \quad\left\|2^{-n} T\left(2^{n} x\right)-U(x)\right\| \leq \delta\|x\| .
$$

Given $\eta>0$, choose $\delta<\eta /\|x\|$ and we have

$$
\left\|\frac{T\left(2^{n} x\right)}{2^{n}}-U(x)\right\| \leq \eta \quad \text { for } n \geq m .
$$

By Lemmas 3.4, 3.5, and the fact that $T(0)=0$, we find that:

$$
\begin{gathered}
V:=U^{-1} T: X \rightarrow X \text { is a surjective } \varepsilon \text {-isometry with } V(0)=0, \\
x=\lim _{n \rightarrow \infty} 2^{-n} V\left(2^{n} x\right) \quad \forall x \in X .
\end{gathered}
$$

Now choose a mapping $V^{-1}: X \rightarrow X$ as follows. Put $V^{-1}(0)=0$, and for $x \in X$ with $x \neq 0$, let $V^{-1}(x)$ be any point $y \in X$ with $V(y)=x$. Then clearly $V^{-1}$ has the following properties:

$$
\begin{gathered}
V^{-1}: X \rightarrow X \text { is an } \varepsilon \text {-isometry with } V^{-1}(0)=0, \\
V\left(V^{-1}(x)\right)=x \quad \forall x \in X .
\end{gathered}
$$

For $x \in X$, by (3.11), (3.12), and (3.13), we have

$$
\begin{aligned}
\left\|2^{-n} V^{-1}\left(2^{n} x\right)-x\right\| & =2^{-n}\left\|V^{-1}\left(2^{n} x\right)-2^{n} x\right\| \\
& \leq 2^{-n}\left(\left\|2^{n} x-V\left(2^{n} x\right)\right\|+\varepsilon\right) \\
& =\left\|x-2^{-n} V\left(2^{n} x\right)\right\|+2^{-n} \varepsilon,
\end{aligned}
$$

so that

$$
x=\lim _{n \rightarrow \infty} 2^{-n} V^{-1}\left(2^{n} x\right) \quad \forall x \in X .
$$


We now use Lemma 3.1 to approximate the $\varepsilon$-isometry $V$ as follows. For each integer $k>0$ let a continuous mapping $V_{k}: X \rightarrow X$ be chosen so that

$$
\left\|V_{k}(x)-V(x)\right\|<\left(1+k^{-1}\right) \varepsilon .
$$

LEMmA 3.6. Let $y \in X$ and $h$ in the dual space $X^{*}$ be chosen so that $\|y\|=1$ and $\|h\|=h(y)=1$. Then $h(V(\beta y)) \geq \beta-4 \varepsilon$ for each $\beta>0$.

Proof. Since $\|h\|=1=\|y\|$, we use (3.11), (3.12), (3.13), and (3.16) to obtain

$$
\begin{aligned}
h\left(-V_{k}\left(2^{-n} V^{-1}\right.\right. & \left.\left.\left(2^{n} \beta y\right)\right)+2^{n} \beta y\right) \leq\left\|V_{k}\left(2^{-n} V^{-1}\left(2^{n} \beta y\right)\right)-2^{n} \beta y\right\| \\
& \leq\left\|V\left(2^{-n}\left(V^{-1}\left(2^{n} \beta y\right)\right)\right)-2^{n} \beta y\right\|+\left(1+k^{-1}\right) \varepsilon \\
& =\left\|V\left(2^{-n}\left(V^{-1}\left(2^{n} \beta y\right)\right)\right)-V\left(V^{-1}\left(2^{n} \beta y\right)\right)\right\|+\left(1+k^{-1}\right) \varepsilon \\
& \leq\left\|2^{-n} V^{-1}\left(2^{n} \beta y\right)-V^{-1}\left(2^{n} \beta y\right)\right\|+\left(2+k^{-1}\right) \varepsilon \\
& =\left(1-2^{-n}\right)\left\|V^{-1}\left(2^{n} \beta y\right)\right\|+\left(2+k^{-1}\right) \varepsilon \\
& \leq\left(1-2^{-n}\right)\left(\left\|2^{n} \beta y\right\|+\varepsilon\right)+\left(2+k^{-1}\right) \varepsilon \\
& =\left(2^{n}-1\right) \beta+\left(3+k^{-1}-2^{-n}\right) \varepsilon .
\end{aligned}
$$

Since $h$ is linear and $h(y)=1$ we have $h\left(V_{k}\left(2^{-n} V^{-1}\left(2^{n} \beta y\right)\right)\right) \geq \beta-\left(3+k^{-1}-2^{-n}\right) \varepsilon$. Now we use (3.15) and the fact that $h$ and $V_{k}$ are both continuous, so that when $n \rightarrow \infty$, we obtain

$$
h\left(V_{k}(\beta y)\right) \geq \beta-\left(3+k^{-1}\right) \varepsilon .
$$

Therefore, by (3.16) and the fact that $\|h\|=1$, we have $h(V(\beta y)) \geq h\left(V_{k}(\beta y)\right)-$ $\left(1+k^{-1}\right) \varepsilon$, so that $h(V(\beta y)) \geq \beta-\left(4+2 k^{-1}\right) \varepsilon$. Since this is true for each integer $k>0$, Lemma 3.6 follows.

LEMMA 3.7. For each $x \in X,\|x-V(x)\| \leq 5 \varepsilon$.

Proof. Denote the closed ball in $X$ with center at zero and radius 1 by $B$. Let $x \in X$ be chosen. Take $y \in X$ with $\|y\|=1$ and such that $x-V(x)$ is an element of the halfray starting from the origin and containing $y$. Thus, $x-V(x)=\omega y$, where $\omega \geq 0$. Next, choose $u \in X$ such that $y-u$ is a half-tangent to $B$ at $y$, that is,

$$
\frac{\|y-\delta u\|-1}{\delta} \longrightarrow 0 \quad \text { as } \delta \rightarrow 0_{+},
$$

and also that

$$
x=\mu y+\theta u
$$

for suitable real numbers $\mu$ and $\theta$, where $\theta \geq 0$. Thus, $V(x)$ has the form

$$
V(x)=v y+\theta u
$$

with $v=\mu-\omega \in \mathbb{R}$. We note that

$$
x-V(x)=(\mu-v) y \quad \text { with } \mu-v=\omega \geq 0 .
$$


Denote by $L$ the linear subspace generated by $u$ and $y$. The line through $y$ and $y-u$ is a tac-line of $B$. Thus, the linear functional $k: L \rightarrow \mathbb{R}$ defined by $k(\xi y+\eta u)=\xi$ for $\xi$ and $\eta \in \mathbb{R}$, is of norm one. By the Hahn-Banach theorem, we can extend $k$ to a linear functional $h: X \rightarrow \mathbb{R}$ which also has norm one. Now $\|h\|=h(y)=k(y)=\|y\|=1$, so by Lemma 3.6 we have $h(V(\beta y)) \geq \beta-4 \varepsilon$ with $\beta>0$. Since $V$ is an $\varepsilon$-isometry, we have

$$
\begin{aligned}
\|\beta y-x\| & \geq\|V(\beta y)-V(x)\|-\varepsilon \geq h(V(\beta y)-V(x))-\varepsilon \\
& =h(V(\beta y))-h(V(x))-\varepsilon .
\end{aligned}
$$

Using (3.21) and noting that $h(u)=k(u)=0$, we obtain the inequality

$$
\|\beta y-x\| \geq \beta-5 \varepsilon-h(v y+\theta u)=\beta-v-5 \varepsilon \text { for each } \beta>0 .
$$

On the other hand, by (3.20) for $\beta>\mu$, we have

$$
\|\beta y-x\|=\|\beta y-\mu y-\theta u\|=(\beta-\mu)\left\|y-\left(\frac{\theta}{\beta-\mu}\right) u\right\| .
$$

By (3.19), given any $\eta>0$, we may choose $\beta=\beta$ ( $\eta$ ) so large that $\|\beta y-x\| \leq \beta-\mu+\eta$. Using (3.24), we obtain $\beta-\mu \geq\|\beta y-x\|-\eta \geq \beta-v-5 \varepsilon-\eta$, or $0 \geq \mu-v-5 \varepsilon-\eta$. Since $\eta$ may be chosen arbitrarily small, we have $\mu-v \leq 5 \varepsilon$, so, from (3.22), since $\|y\|=1$, we see that $\|x-V(x)\| \leq 5 \varepsilon$. Now, by definition, $V=U^{-1} T$, and, since $U$ is an isometry, we conclude that $\|U(x)-T(x)\| \leq 5 \varepsilon$.

COROLLARY 3.8. Under the hypotheses of Theorem 3.3, suppose that in addition $T$ is continuous. Then the above inequality may be improved as $\|U(x)-T(x)\| \leq 3 \varepsilon$.

Proof. When $T$ is continuous, we can eliminate the approximations $V_{k}$ and use $V$ directly instead. Then the conclusion of Lemma 3.6 can be changed to $h(V(\beta y)) \geq$ $\beta-2 \varepsilon$, and the corollary follows.

It remained for Gevirtz [12] to at last prove the following result which establishes the stability of isometries between arbitrary Banach spaces.

THEOREM 3.9. Given real Banach spaces $X$ and $Y$, let $f: X \rightarrow Y$ be a surjective $\varepsilon$ isometry. Then there exists a surjective isometry $U: X \rightarrow Y$ for which $\|f(x)-U(x)\| \leq 5 \varepsilon$.

The main part of the proof is to demonstrate the following result.

THEOREM 3.10 (Gevirtz [12]). If $f: X \rightarrow Y$ is a surjective $\varepsilon$-isometry, then, for any pair $x_{0}, x_{1} \in X$, we have

$$
\left\|f\left(\frac{x_{0}+x_{1}}{2}\right)-\frac{f\left(x_{0}\right)+f\left(x_{1}\right)}{2}\right\| \leq 10 \sqrt{\varepsilon\left\|x_{0}-x_{1}\right\|}+20 \varepsilon .
$$

Proof. The idea of the proof was to "epsilonize" the method of Vogt which was used above in demonstrating Theorems 1.1 and 1.2. From Bourgin's $\delta$-onto idea, Gevirtz developed the following definition.

Definition 3.11. Given $f: X \rightarrow Y$, a mapping $F: Y \rightarrow X$ for which

$$
\|f F(y)-y\| \leq \delta
$$


for all $y \in Y$ is called a $\delta$-inverse of $f$ and $f$ is called $\delta$-onto if it has a $\delta$-inverse. Also, the term $(\delta, \varepsilon)$-isometry is an abbreviation for " $\delta$-onto $\varepsilon$-isometry."

We will need the following lemmas.

LEMMA 3.12. If $f: X \rightarrow Y$ is a $(\delta, \varepsilon)$-isometry and $F$ is a $\delta$-inverse of $f$, then $F$ is a $(\delta+\varepsilon, 2 \delta+\varepsilon)$-isometry.

Proof. $\|F f(x)-x\| \leq\|f(F f(x))-f(x)\|+\varepsilon \leq \delta+\varepsilon$ by (3.27), so $F$ is $(\delta+\varepsilon)$-onto. For $y_{0}$ and $y_{1} \in Y$ we have by (3.27) that $\left\|f F\left(y_{j}\right)-y_{j}\right\| \leq \delta, j=0,1$. Since $f$ is an $\varepsilon$-isometry, $\left|\left\|f F\left(y_{0}\right)-f F\left(y_{1}\right)\right\|-\left\|F\left(y_{0}\right)-F\left(y_{1}\right)\right\|\right| \leq \varepsilon$.

Hence,

$$
\begin{aligned}
& \left|\left\|F\left(y_{0}\right)-F\left(y_{1}\right)\right\|-\left\|y_{0}-y_{1}\right\|\right| \\
& \quad \leq\left|\left\|f F\left(y_{0}\right)-f F\left(y_{1}\right)\right\|-\left\|y_{0}-y_{1}\right\|\right|+\varepsilon \\
& \quad \leq\left|\left\|f F\left(y_{0}\right)-y_{0}\right\|+\left\|y_{1}-f F\left(y_{1}\right)\right\|+\left\|y_{0}-y_{1}\right\|-\left\|y_{0}-y_{1}\right\|\right|+\varepsilon \leq 2 \delta+\varepsilon,
\end{aligned}
$$

so that $F$ is a $(2 \delta+\varepsilon)$-isometry.

LEMMA 3.13. Let $f_{1}: X \rightarrow Y$ be a $\left(\delta_{1}, \varepsilon_{1}\right)$-isometry, let $Z$ be another Banach space and let $f_{2}: Y \rightarrow Z$ be a $\left(\delta_{2}, \varepsilon_{2}\right)$-isometry. Then $f_{2} f_{1}: X \rightarrow Z$ is a $\left(\delta_{1}+\delta_{2}+\varepsilon_{2}, \varepsilon_{1}+\varepsilon_{2}\right)$-isometry.

Proof. By hypotheses, we have

$$
\begin{gathered}
||\left|f_{2} f_{1}\left(x_{0}\right)-f_{2} f_{1}\left(x_{1}\right)\|-\| f_{1}\left(x_{0}\right)-f_{1}\left(x_{1}\right) \|\right| \leq \varepsilon_{2}, \\
\left|\left\|f_{1}\left(x_{0}\right)-f_{1}\left(x_{1}\right)\right\|-\left\|x_{0}-x_{1}\right\|\right| \leq \varepsilon_{1},
\end{gathered}
$$

so that

$$
||\left|f_{2} f_{1}\left(x_{0}\right)-f_{2} f_{1}\left(x_{1}\right)\|-\| x_{0}-x_{1} \|\right| \leq \varepsilon_{2}+\varepsilon_{1} .
$$

Thus, $f_{2} f_{1}$ is an $\left(\varepsilon_{1}+\varepsilon_{2}\right)$-isometry. Now let $F_{j}$ be a $\delta_{j}$-inverse of $f_{j}, j=1,2$, and let $z$ be any element of $Z$. Since $f_{2}$ is an $\varepsilon_{2}$-isometry, we can apply (3.27), to $f_{2}$ and $f_{1}$, to obtain

$$
\begin{aligned}
\left\|f_{2} f_{1} F_{1} F_{2}(z)-z\right\| & \leq\left\|f_{2} f_{1} F_{1} F_{2}(z)-f_{2} F_{2}(z)\right\|+\left\|f_{2} F_{2}(z)-z\right\| \\
& \leq\left\|f_{1} F_{1} F_{2}(z)-F_{2}(z)\right\|+\varepsilon_{2}+\delta_{2} \leq \delta_{1}+\delta_{2}+\varepsilon_{2} .
\end{aligned}
$$

With the given $x_{0}$ and $x_{1} \in X$, let $y_{0}=f\left(x_{0}\right), y_{1}=f\left(x_{1}\right)$ and put

$$
p=\frac{x_{0}+x_{1}}{2}, \quad q=\frac{y_{0}+y_{1}}{2} .
$$

For the present, we assume that $y_{0} \neq y_{1}$. Since $f$ is surjective it has an inverse, so it is a $(0, \varepsilon)$-isometry. By Lemma 3.12 , its inverse $F$ is an $(\varepsilon, \varepsilon)$-isometry for which

$$
F\left(y_{0}\right)=x_{0}, \quad F\left(y_{1}\right)=x_{1}
$$

We now define sequences $\left(g_{k}\right)_{k \geq 0}$ and $\left(G_{k}\right)_{k \geq 0}$ as follows:

$$
g_{0}(y)=f(2 p-F(y)) \text { for } y \in Y .
$$


By Lemma 3.13, $g_{0}: Y \rightarrow Y$ is a $(2 \varepsilon, 2 \varepsilon)$-isometry, and clearly $g_{0}\left(y_{j}\right)=y_{1-j}$ for $j=0,1$. We define $G_{0}: Y \rightarrow Y$ to be any mapping such that:

$$
G_{0} \text { is a }(4 \varepsilon) \text {-inverse of } g_{0}, \quad G_{0}\left(y_{j}\right)=y_{1-j}, \quad j=0,1 .
$$

For $y \in Y$, let

$$
g_{1}(y)=G_{1}(y)=2 q-y .
$$

Finally, if we are given $g_{0}, \ldots, g_{n}$ and $G_{0}, \ldots, G_{n}$, we define $G_{n+1}$ as any mapping which is a $4^{n+2} \varepsilon$-inverse of $g_{n+1}$ and $G_{n+1}\left(y_{j}\right)=y_{1-j}, j=0,1$, where

$$
g_{n+1}=g_{n-1} g_{n} G_{n-1} \quad(n \geq 1) .
$$

LEMmA 3.14. The sequences $\left(g_{k}\right)$ and $\left(G_{k}\right)$ defined by (3.34), (3.35), (3.36), and (3.37) have the following properties:

$$
\begin{gathered}
g_{k} \text { is a }\left(4^{k+1} \varepsilon, 4^{k+1} \varepsilon\right) \text {-isometry, } \quad g_{k}\left(y_{j}\right)=y_{1-j}, \quad j=0,1, \\
G_{k} \text { is a }\left(4^{k+1} \varepsilon\right) \text {-inverse of } g_{k}, \quad G_{k}\left(y_{j}\right)=y_{1-j}, \quad j=0,1 .
\end{gathered}
$$

Proof. The properties (3.38) and (3.39) are true for $k=0$ and $k=1$, as we have seen. Make the induction assumption that both are true when $0 \leq k \leq n$. By (3.37) we have $g_{n+1}=g_{n-1} g_{n} G_{n-1}$ and we know that $g_{n-1}$ is a $\left(4^{n} \varepsilon, 4^{n} \varepsilon\right)$-isometry and $G_{n-1}$ is a $\left(4^{n} \varepsilon\right.$ )-inverse of $g_{n-1}$. Hence, by Lemmas 3.12 and 3.13, it follows that $g_{n+1}$ is a $\left(4^{n+2} \varepsilon, 4^{n+2} \varepsilon\right)$-isometry. Clearly, $g_{n+1}\left(y_{j}\right)=g_{n-1} g_{n} G_{n-1}\left(y_{j}\right)=y_{1-j}, j=0,1$ so $g_{n+1}$ satisfies (3.38) with $k=n+1$. By definition, $G_{n+1}$ satisfies (3.39) with $k=n+1$, and the induction is complete.

Proof OF TheOrem 3.10. We define a sequence of points $a_{n} \in Y$ recursively by

$$
a_{1}=q=\frac{y_{0}+y_{1}}{2}, \quad a_{n+1}=g_{n-1}\left(a_{n}\right), \quad n \geq 1 .
$$

Put $d=\left\|y_{0}-y_{1}\right\| / 2$. Let $B(y, r)$ denote the closed ball in $Y$ with center $y$ and radius $r>0$. Since $g_{k}$ is a $\left(4^{k+1} \varepsilon\right)$-isometry for $k \geq 0$, when $y \in B\left(y_{0}, r\right)$, we have $\left|\left\|g_{k}(y)-g_{k}\left(y_{0}\right)\right\|-\left\|y-y_{0}\right\|\right| \leq 4^{k+1} \varepsilon$ and therefore $\left\|g_{k}(y)-y_{1}\right\| \leq r+4^{k+1} \varepsilon$, where we have used $g_{k}\left(y_{0}\right)=y_{1}$. Thus, $g_{k}\left(B\left(y_{0}, r\right)\right) \subset B\left(y_{1}, r+4^{k+1} \varepsilon\right)$, and clearly the same is true with $y_{0}$ and $y_{1}$ interchanged. Therefore, we have

$$
g_{k}\left(B\left(y_{j}, r\right)\right) \subset B\left(y_{1-j}, r+4^{k+1} \varepsilon\right), \quad j=0,1 .
$$

Evidently, $a_{1} \in B\left(y_{0}, d\right) \cap B\left(y_{1}, d\right)$. Also $a_{n}=g_{n-2}\left(a_{n-1}\right)=g_{n-2} g_{n-3} \cdots g_{0}\left(a_{1}\right)$, so an induction based on successive applications of (3.41) with $k=0,1, \ldots, n-2$ gives $a_{n} \in B\left(y_{0}, d+4^{n} \varepsilon\right) \cap B\left(y_{1}, d+4^{n} \varepsilon\right) \subset B\left(q, d+4^{n} \varepsilon\right)$ for $n \geq 1$. Now the diameter of this last ball is $2\left(d+4^{n} \varepsilon\right)$, so that

$$
\left\|a_{n}-a_{n-1}\right\| \leq 2\left(d+4^{n} \varepsilon\right), \quad n \geq 2 .
$$

Next we prove by induction that

$$
\left\|g_{n}(y)-y\right\| \geq 2\left\|a_{n}-y\right\|-2\left(4^{n} \varepsilon\right), \quad n \geq 1 .
$$


For $n=1$, we have by (3.36) and (3.40) that $g_{1}(y)=2 q-y=2 a_{1}-y$, so that, $\left\|g_{1}(y)-y\right\|=\left\|2 a_{1}-2 y\right\|=2\left\|a_{1}-y\right\| \geq 2\left\|a_{1}-y\right\|-2(4 \varepsilon)$, which verifies (3.43) for $n=1$. Assuming that (3.43) is true, we shall show that it holds when $n$ is replaced by $n+1$. Using the recursion formula (3.37) and Lemma 3.14, we obtain

$$
\begin{aligned}
\left\|g_{n+1}(y)-y\right\| & =\left\|g_{n-1} g_{n} G_{n-1}(y)-y\right\| \\
& =\left\|g_{n-1} g_{n} G_{n-1}(y)-g_{n-1} G_{n-1}(y)+g_{n-1} G_{n-1}(y)-y\right\| \\
& \geq\left\|g_{n-1} g_{n} G_{n-1}(y)-g_{n-1} G_{n-1}(y)\right\|-\left\|g_{n-1} G_{n-1}(y)-y\right\| \\
& \geq\left\|g_{n-1} g_{n} G_{n-1}(y)-g_{n-1} G_{n-1}(y)\right\|-4^{n} \varepsilon \\
& \geq\left\|g_{n} G_{n-1}(y)-G_{n-1}(y)\right\|-2\left(4^{n} \varepsilon\right) .
\end{aligned}
$$

Now use the induction hypothesis with $y$ replaced by $G_{n-1}(y)$ to find that

$$
\begin{aligned}
\left\|g_{n+1}(y)-y\right\| & \geq 2\left\|a_{n}-G_{n-1}(y)\right\|-4^{n+1} \varepsilon \\
& \geq 2\left(\left\|g_{n-1}\left(a_{n}\right)-g_{n-1} G_{n-1}(y)\right\|-4^{n} \varepsilon\right)-4^{n+1} \varepsilon \\
& \geq 2\left(\left\|a_{n+1}-y\right\|-2\left(4^{n} \varepsilon\right)\right)-4^{n+1} \varepsilon=2\left\|a_{n+1}-y\right\|-2\left(4^{n+1} \varepsilon\right),
\end{aligned}
$$

so the induction is complete for (3.43).

By (3.40) and (3.43), we have $\left\|a_{n+1}-a_{n}\right\|=\left\|g_{n-1}\left(a_{n}\right)-a_{n}\right\| \geq 2\left\|a_{n}-a_{n-1}\right\|-$ $2\left(4^{n-1} \varepsilon\right)$, for $n \geq 1$. We replace $n$ by $n-1$ to get $\left\|a_{n}-a_{n-1}\right\| \geq 2\left\|a_{n-1}-a_{n-2}\right\|-$ $2\left(4^{n-2} \varepsilon\right)$ and again $\left\|a_{n-1}-a_{n-2}\right\| \geq 2\left\|a_{n-2}-a_{n-3}\right\|-2\left(4^{n-3} \varepsilon\right)$. Substituting the last inequality into the preceding one, we have

$$
\left\|a_{n}-a_{n-1}\right\| \geq 2^{2}\left\|a_{n-2}-a_{n-3}\right\|-4^{n-1} \varepsilon .
$$

By induction, we arrive at the inequality

$$
\left\|a_{n}-a_{n-1}\right\| \geq 2^{n-2}\left\|a_{2}-a_{1}\right\|-4^{n-1} \varepsilon, \text { for } n \geq 2 .
$$

Now, by (3.42),

$$
\left\|a_{n}-a_{n-1}\right\| \leq 2\left(d+4^{n} \varepsilon\right)
$$

and we have

$$
\left\|a_{2}-a_{1}\right\| \leq 2^{2-n}\left(2 d+2\left(4^{n} \varepsilon\right)+4^{n-1} \varepsilon\right) \text { for } n \geq 2 .
$$

Changing $n$ to $n+2$, now for $n \geq 0$, we find that

$$
\left\|a_{2}-a_{1}\right\| \leq 2\left(d 2^{-n}+18 \varepsilon 2^{n}\right) .
$$

By (3.34) and (3.40), $a_{1}=q$ and $a_{2}=g_{0}(q)=f(2 p-F(q))$. Hence,

$$
\begin{aligned}
\left\|a_{2}-a_{1}\right\| & =\|f(2 p-F(q))-q\|=\|f(2 p-F(q))-f F(q)\| \geq\|2 p-2 F(q)\|-\varepsilon \\
& =2\|p-F(q)\|-\varepsilon \geq 2(\|f(p)-f F(q)\|-\varepsilon)-\varepsilon=2\|f(p)-q\|-3 \varepsilon .
\end{aligned}
$$

Thus, by (3.50), for $n \geq 0$ we have

$$
\|f(p)-q\| \leq d 2^{-n}+18 \varepsilon 2^{n}+2 \varepsilon .
$$

We consider two cases according to whether or not $d>18 \varepsilon$. 
CASE $1(d>18 \varepsilon)$. Let the real number $t$ be defined by $d 2^{-t}=18 \varepsilon 2^{t}$, that is, $t=$ $\log _{4}(d / 18 \varepsilon)>0$. Let $n$ be the greatest integer in $t$. Then $2^{-n}<2 \cdot 2^{-t}$ so by (3.52)

$$
\|f(p)-q\| \leq 2 d \cdot 2^{-t}+18 \varepsilon 2^{t}+2 \varepsilon=3 d \cdot 2^{-t}+2 \varepsilon,
$$

by the definition of $t$. Note also from this definition that

$$
d^{2} \cdot 2^{-2 t}=18 \varepsilon d, \quad d \cdot 2^{-t}=\sqrt{18 \varepsilon d} .
$$

Hence,

$$
\|f(p)-q\| \leq 3 \sqrt{18 \varepsilon d}+2 \varepsilon .
$$

Since $f$ is an $\varepsilon$-isometry and $d>18 \varepsilon$, we have

$$
\left\|x_{0}-x_{1}\right\| \geq\left\|y_{1}-y_{0}\right\|-\varepsilon=2 d-\varepsilon>2 d-\frac{d}{18}=\frac{35 d}{18},
$$

and so

$$
18 d \leq\left(\frac{18^{2}}{35}\right)\left\|x_{0}-x_{1}\right\|
$$

Thus, in Case 1, we find that

$$
\|f(p)-q\| \leq 10 \sqrt{\varepsilon\left\|x_{0}-x_{1}\right\|}+2 \varepsilon .
$$

CASE $2(d \leq 18 \varepsilon)$. This case covers the situation, where $y_{0}=y_{1}$ which was excluded earlier. Here

$$
\left\|y_{0}-y_{1}\right\|=2 d \leq 36 \varepsilon
$$

so that

$$
\left\|x_{0}-x_{1}\right\| \leq 37 \varepsilon .
$$

Thus, $\left\|x_{j}-p\right\| \leq 19 \varepsilon$ and $\left\|y_{j}-f(p)\right\| \leq 20 \varepsilon, j=0,1$. Since

$$
\begin{gathered}
q=\frac{y_{0}+y_{1}}{2}, \\
\|f(p)-q\|=\left\|\frac{f(p)}{2}-\frac{y_{0}}{2}+\frac{f(p)}{2}-\frac{y}{2}\right\| \leq\left\|\frac{f(p)-y_{0}}{2}\right\|+\left\|\frac{f(p)-y_{1}}{2}\right\| \leq 20 \varepsilon .
\end{gathered}
$$

Therefore, in either Case 1 or Case 2, we have demonstrated the inequality

$$
\|f(p)-q\| \leq 10 \sqrt{\varepsilon\left\|x_{0}-x_{1}\right\|}+20 \varepsilon,
$$

which by (3.32) is the required inequality (3.26) of Theorem 3.10.

Proof of TheOrem 3.9. We may assume, without loss of generality, that $f(0)=$ 0 . Put $x_{0}=2 x, x_{1}=0$ in (3.26) to get

$$
\left\|\frac{f(2 x)}{2}-f(x)\right\| \leq 10 \sqrt{2}(\varepsilon\|x\|)^{1 / 2}+20 \varepsilon .
$$

We prove by induction that

$$
\left\|\frac{f\left(2^{n} x\right)}{2^{n}}-f(x)\right\| \leq 10 \sqrt{2}\left(\sum_{k=0}^{n-1} 2^{-k / 2}\right)(\varepsilon\|x\|)^{1 / 2}+20 \varepsilon \sum_{k=0}^{n-1} 2^{-k} .
$$


Hence,

$$
\left\|2^{-n} f\left(2^{n} x\right)-f(x)\right\| \leq 20(1+\sqrt{2})(\varepsilon\|x\|)^{1 / 2}+40 \varepsilon .
$$

By replacing $x$ with $2^{m} x$ in (3.65) and dividing the result by $2^{m}$, we get

$$
\left\|2^{-m-n} f\left(2^{m+n} x\right)-2^{-m} f\left(2^{m} x\right)\right\| \leq 20(1+\sqrt{2}) 2^{-m / 2}(\varepsilon\|x\|)^{1 / 2}+40 \varepsilon 2^{-m},
$$

which shows that $\left(2^{-n} f\left(2^{n} x\right)\right)$ is a Cauchy sequence. Since $Y$ is a Banach space, there exists $U: X \rightarrow Y$ such that

$$
U(x)=\lim _{n \rightarrow \infty} \frac{f\left(2^{n} x\right)}{2^{n}} \text { for each } x \in X .
$$

Moreover, if $n \rightarrow \infty$ in (3.65), we obtain

$$
\|U(x)-f(x)\| \leq 20(1+\sqrt{2})(\varepsilon\|x\|)^{1 / 2}+40 \varepsilon .
$$

It follows immediately that $\|U(x)-f(x)\| /\|x\| \rightarrow 0$ uniformly as $\|x\| \rightarrow 0$ uniformly, and we know that $f(0)=U(0)=0$. In order to apply Theorem 3.3, it remains only to show that $U$ is an isometry. Since $f$ is an $\varepsilon$-isometry on $X$, we have

$$
|| 2^{-n} f\left(2^{n} x_{0}\right)-2^{-n} f\left(2^{n} x_{1}\right)\|-\| x_{0}-x_{1} \| \mid \leq \frac{\varepsilon}{2^{n}}
$$

and in the limit as $n \rightarrow \infty$ we get $\left\|U\left(x_{0}\right)-U\left(x_{1}\right)\right\|=\left\|x_{0}-x_{1}\right\|$ for every pair of points $x_{0}, x_{1} \in X$. Now all the requirements of the Gruber Theorem 3.3 above are satisfied and we conclude that $U$ is surjective and that $\|U(x)-f(x)\| \leq 5 \varepsilon$ for all $x \in X$.

Comments. Gruber [13] raised the question whether his bounds $3 \varepsilon$ for $\| T(x)-$ $U(x) \|$ when the $\varepsilon$-isometry $T$ is continuous and $5 \varepsilon$ in general could be improved. He showed that if, in Theorem 3.3 above, the space $X$ is Euclidean, then the bounds $3 \varepsilon$ and $5 \varepsilon$ may be replaced by $3 \varepsilon / \sqrt{2}$ and $5 \varepsilon / \sqrt{2}$, respectively.

Recently, Omladič and Šemrl [18] have shown that indeed these bounds can be reduced. Their main result is the following theorem.

TheOrem 3.15. Let $X$ and $Y$ be real Banach spaces. Given $\varepsilon>0$, let $f: X \rightarrow Y$ be a surjective $\varepsilon$-isometry with $f(0)=0$. Then there exists a surjective isometry $U: X \rightarrow Y$ such that

$$
\|f(x)-U(x)\| \leq 2 \varepsilon \quad \forall x \in X .
$$

These authors gave examples to show that $2 \varepsilon$ is the best possible result. Their simplest example is this. Define $f: \mathbb{R} \rightarrow \mathbb{R}$ by

$$
f(t)= \begin{cases}-3 t & \text { when } 0 \leq t \leq \frac{1}{2} \\ t-1 & \text { otherwise }\end{cases}
$$

It is easy to see that $f$ is a 1-isometry, $U(t)=t$ for this function, and also

$$
\max \{|f(t)-U(t)|: t \in \mathbb{R}\}=2 \text {. }
$$


Thus, the inequality is sharp. They also constructed an example of a homeomorphic mapping $f: \mathbb{R}^{2} \rightarrow \mathbb{R}^{2}$ which is a 1-isometry and such that 2 cannot be replaced by a smaller constant in (3.70). Thus, the sharpness persists even for homeomorphisms.

Skof [21] has given a different approach to the stability problem for isometric mappings between real normed spaces $X$ and $E$, in which neither completeness of the spaces nor surjectivity of the mappings is required. Also, the methods used are elementary.

SKOF'S THEOREM. Let $T: X \rightarrow E$ with $T(0)=0$ satisfy the conditions: (1) $T$ is a $\delta$ isometry for some $\delta>0$, and (2) for each $x \in X$, both $T(-x)$ and $T(2 x)$ are scalar multiples of $T(x)$. Let $E_{0}$ denote the linear hull of $T(X) \in E$. Then there exists an isometry $U: X \rightarrow E_{0}$ which satisfies the inequality $\|T(x)-U(x)\|<36 \delta$ for all $x \in X$ and is given by the formula $U(x)=\lim _{n \rightarrow \infty} 2^{-n} T\left(2^{n} x\right)$.

The proof depends upon another theorem by the same author which establishes the stability of the alternative Cauchy equation: $\|\varphi(x+y)\|=\|\varphi(x)+\varphi(y)\|$. In general, neither $T$ nor $U$ need to be linear. For instance, recall the example given by Baker [1] which was mentioned after Theorem 3.15. Let $X=\mathbb{R}^{2}$ with the usual norm, let $E$ be $\mathbb{R}^{3}$ but with the norm $(x, y, z)=\max \left[\sqrt{x^{2}+y^{2}},|z|\right]$ and $f: X \rightarrow E$ be the mapping given by Baker in the second example cited above. Then $f$ is an isometry which is homogeneous and nonlinear. Clearly, this mapping $f$ satisfies the conditions of Skof's theorem. Here $T=U=f$.

$\varepsilon$-ISOMETRIES ON BOUNDED SETS. Swain [23] considered $\varepsilon$-isometries on bounded metric spaces and proved the following result.

THEOREM 3.16. Let $M$ be a subset of a compact metric space $(E, d)$ and let $\eta>0$ be given. Then there exists an $\varepsilon>0$ such that, if $T$ is any $\varepsilon$-isometry of $M$ into a subset of $E$, there is an isometry $U: M \rightarrow E$ such that $d(U(x), T(x))<\eta$ for all $x \in M$.

The same author also gave an example to show that even for $\varepsilon$-isometries $T$ on convex subsets $M$ of $\mathbb{R}^{2}$ into $\mathbb{R}^{2}$ there is no universal constant $k$ such that $d(T(x), U(x)) \leq$ $k \varepsilon$ for $x \in M$ and for all such subsets $M$. The difficulty arises here for long thin sets $M$.

The problem of $\varepsilon$-isometries on bounded subsets of $\mathbb{R}^{n}$ was studied by Fickett [9]. He proved the following two stability theorems, the first depending on the size of the subset in question and the second on the shape of the subset.

For $t \geq 0$, define $K_{0}(t)=K_{1}(t)=t, K_{2}(t)=3(3 t)^{1 / 2}, K_{j}(t)=27 t^{m(j)}$, where $m(j)=$ $1 / 2^{j-1}$ for $j \geq 3$.

THEOREM 3.17. Let $S$ be a bounded subset of $\mathbb{R}^{n}$ with diameter $\delta(S)$, and let $T: S \rightarrow$ $\mathbb{R}^{n}$ be an $\varepsilon$-isometry for some $\varepsilon \geq 0$. Suppose that $3 K_{n}(\varepsilon / \delta(s)) \leq 1$. Then there exists an isometry $U: S \rightarrow \mathbb{R}^{n}$ such that $d(T(x), U(x)) \leq \delta(S) K_{n+1}(\varepsilon / \delta(S))$ for all $x \in S$.

The next theorem of Fickett illustrates the "difficulty" referred to above for thin sets. He defines the minimum thickness of a bounded set $S$ to be the infimum of those $d>0$ such that $S$ will fit between two parallel hyper-planes a distance $d$ apart.

THEOREM 3.18. Consider a set $S \subset \mathbb{R}^{n}$ with diameter $\delta(S)<\infty$ and with minimum thickness $h$. For some $\varepsilon \geq 0$, let $T: S \rightarrow \mathbb{R}^{n}$ be an $\varepsilon$-isometry. Then there exists an 
isometry $U: S \rightarrow \mathbb{R}^{n}$ such that for all $x \in S$,

$$
d(T(x), U(x)) \leq c_{n} \varepsilon\left(\frac{\delta(S)}{h}\right)^{2 n+4}, \quad \text { where } c_{n}=2^{\left(n^{2} / 4\right)+3 n+22} .
$$

Fickett used the first of these results to prove a theorem concerning a conjecture of Ulam on the invariance of measure in the Hilbert cube.

Jung [16] modified the method (3.8) slightly to prove the stability of isometries on restricted but unbounded domains, while Fickett investigated the stability problems of isometries on bounded sets.

\section{REFERENCES}

[1] J. A. Baker, Isometries in normed spaces, Amer. Math. Monthly 78 (1971), 655-658. MR 44\#4490. Zbl 214.12704.

[2] S. Banach, Théorie des Opérations Linéaires [Theory of Linear Operators], Éditions Jacques Gabay, Sceaux, 1993 (French), Reprint of the 1932 original. MR 97d:01035. Zbl 005.20901.

[3] D. G. Bourgin, Approximate isometries, Bull. Amer. Math. Soc. 52 (1946), 704-714. MR 8,157f. Zbl 060.26405.

[4] - Approximately isometric and multiplicative transformations on continuous function rings, Duke Math. J. 16 (1949), 385-397. MR 11,115e. Zbl 033.37702.

[5] _ Two-dimensional E-isometries, Trans. Amer. Math. Soc. 244 (1978), 85-102. MR 80d:46027. Zbl 412.46011.

[6] R. D. Bourgin, Approximate isometries on finite dimensional Banach spaces, Trans. Amer. Math. Soc. 207 (1975), 309-328. MR 51\#6366. Zbl 327.46023.

[7] Z. Charzyński, Sur les transformations isométriques des espaces du type ( $F)$, Studia Math. 13 (1953), 94-121 (French). MR 15,38f. Zbl 051.08504.

[8] M. M. Day, Normed Linear Spaces, Ergebnisse der Mathematik und ihrer Grenzgebiete. Neue Folge. Heft 21. Reihe: Reelle Funktionen, Springer-Verlag, Berlin, Göttingen, Heidelberg, 1958. MR 20\#1187. Zbl 082.10603.

[9] J. W. Fickett, Approximate isometries on bounded sets with an application to measure theory, Studia Math. 72 (1982), no. 1, 37-46. MR 84d:28019. Zbl 488.28006.

[10] T. Figiel, On nonlinear isometric embeddings of normed linear spaces, Bull. Acad. Polon. Sci. Sér. Sci. Math. Astronom. Phys. 16 (1968), 185-188. MR 37\#6734. Zbl 155.18301.

[11] P. Fischer and G. Muszély, On some new generalizations of the functional equation of Cauchy, Canad. Math. Bull. 10 (1967), 197-205. MR 35\#7025. Zbl 157.22303.

[12] J. Gevirtz, Stability of isometries on Banach spaces, Proc. Amer. Math. Soc. 89 (1983), no. 4, 633-636. MR 85d:46019. Zbl 561.46012.

[13] P. M. Gruber, Stability of isometries, Trans. Amer. Math. Soc. 245 (1978), 263-277. MR 81a:41053. Zbl 393.41020.

[14] D. H. Hyers and S. M. Ulam, On approximate isometries, Bull. Amer. Math. Soc. 51 (1945), 288-292. MR 7,123f. Zbl 060.26404.

[15]___ Approximate isometries of the space of continuous functions, Ann. of Math. (2) 48 (1947), 285-289. MR 8,588b. Zbl 029.36701.

[16] S. M. Jung, Hyers-Ulam-Rassias stability of isometries on restricted domains, submitted.

[17] S. Mazur and S. Ulam, Sur les transformations isometriques d'espaces vectoriels normes, C. R. Acad. Sci. 194 (1932), 946-948 (French), See also [2, page 166]. Zbl 004.02103.

[18] M. Omladič and P. Šemrl, On nonlinear perturbations of isometries, Math. Ann. 303 (1995), no. 4, 617-628. MR 96k:46017. Zbl 836.46014.

[19] S. Rolewicz, A generalization of the Mazur-Ulam theorem, Studia Math. 31 (1968), 501505. MR 39\#751. Zbl 185.20604. 
[20] I. J. Schoenberg, Metric spaces and completely monotone functions, Ann. of Math. (2) 39 (1938), 811-841. Zbl 019.41503.

[21] F. Skof, Sulle $\delta$-isometrie negli spazi normati, Rend. Mat. (7) 10 (1990), 853-866 (Italian).

[22] M. H. Stone, Applications of the theory of Boolean rings to general topology, Trans. Amer. Math. Soc. 41 (1937), no. 3, 375-481. CMP 1501 905. Zbl 017.13502.

[23] R. L. Swain, Approximate isometries in bounded spaces, Proc. Amer. Math. Soc. 2 (1951), 727-729. MR 13,373a. Zbl 044.19603.

[24] A. Vogt, Maps which preserve equality of distance, Studia Math. 45 (1973), 43-48. MR 48\#12001. Zbl 246.46010.

[25] J. von Neumann and I. J. Schoenberg, Fourier integrals and metric geometry, Trans. Amer. Math. Soc. 50 (1941), 226-251. MR 3,37g. Zbl 028.41002.

[26] R. Wobst, Isometrien in metrischen Vektorräumen, Studia Math. 54 (1975), no. 1, 41-54 (German). MR 54\#5786. Zbl 318.46019.

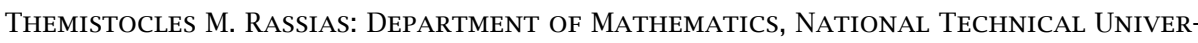
SITY OF ATHENS, ZOGRAFOU CAMPUS, 15780 ATHENS, GREECE.

E-mail address: trassias@math.ntua.gr 


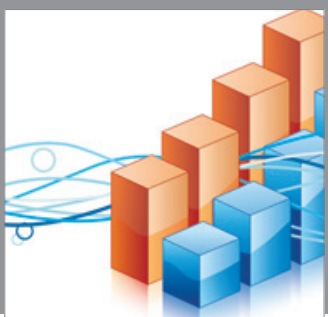

Advances in

Operations Research

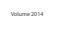

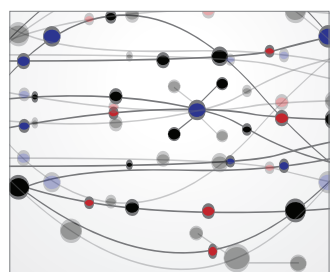

\section{The Scientific} World Journal
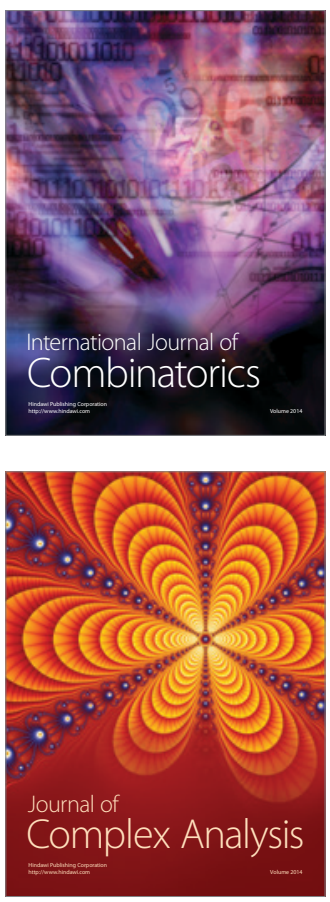

International Journal of

Mathematics and

Mathematical

Sciences
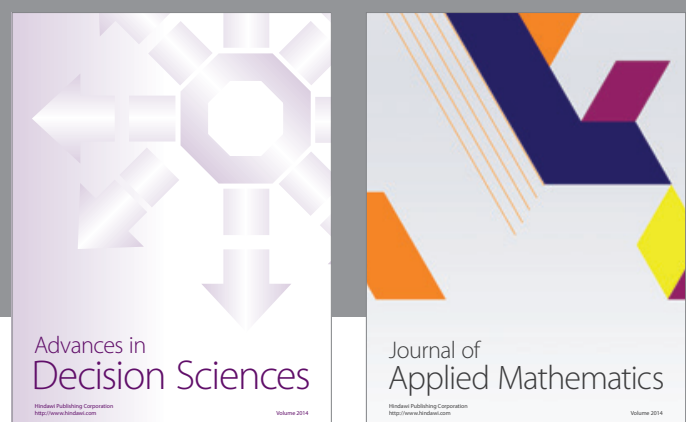

Journal of

Applied Mathematics
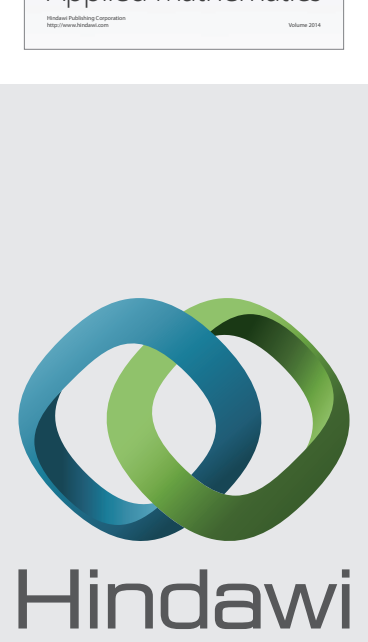

Submit your manuscripts at http://www.hindawi.com
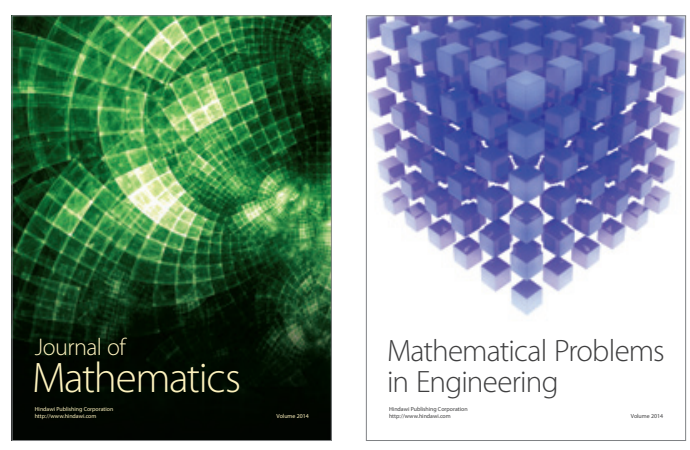

Mathematical Problems in Engineering
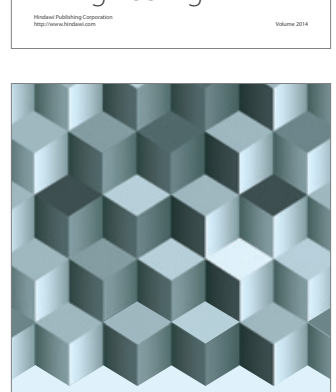

Journal of

Function Spaces
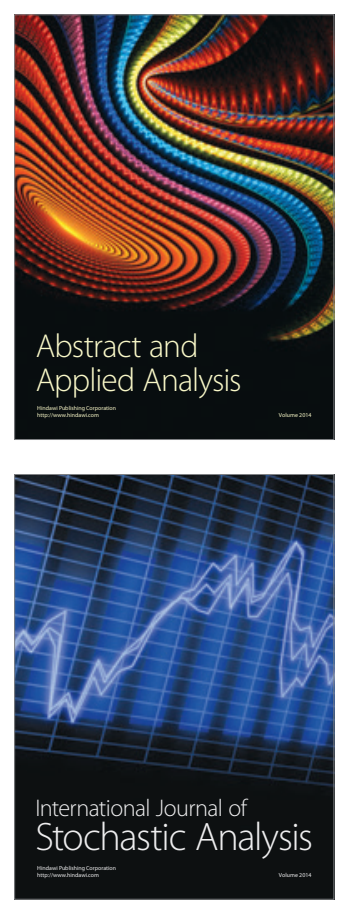

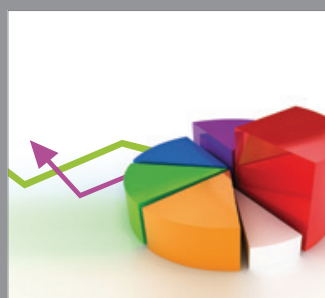

ournal of

Probability and Statistics

Promensencen
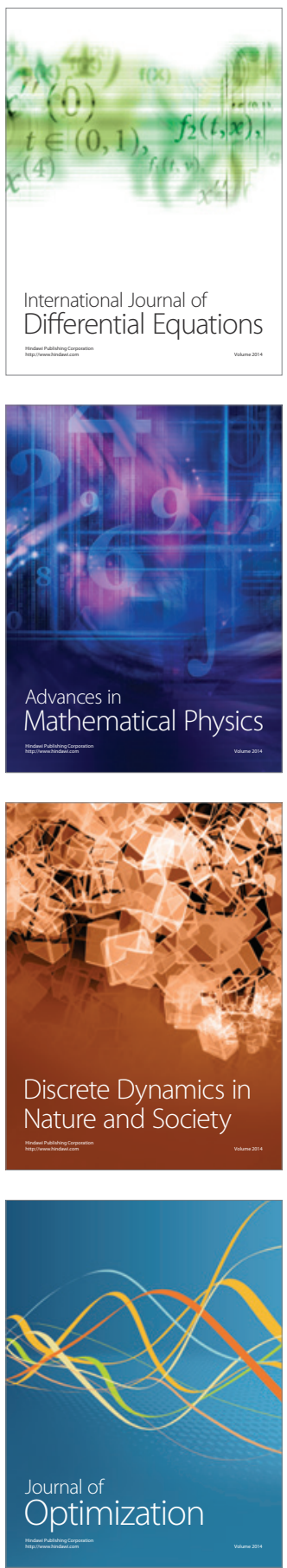